\title{
Diagnosis of asthmatic pneumonia in children by lung ultrasound vs. chest X-ray: an updated systematic review and meta-analysis
}

\author{
Qi Ru¹, LanLan Liư ${ }^{2}$ Xiaoyun Dong ${ }^{1}$ \\ ${ }^{1}$ Department of Ultrasound, Qilu Hospital (Qingdao), Cheeloo College of Medicine, Shandong University, Shandong Province, Qingdao, \\ China \\ ${ }^{2}$ Special Inspection Section, Qingdao Women and Children's Hospital, Shandong Province, Qingdao, China \\ Adv Dermatol Allergol 2023; XL (1): 28-34 \\ DOI: https://doi.org/10.5114/ada.2021.108441
}

\begin{abstract}
Introduction: Chest X-ray (CXR) is used as the standard diagnostic method in lung diseases, especially in pneumonia, but unfortunately, despite the high risk of receiving radiation, it also has a high false negative rate. Therefore, some researchers recommend ultrasound to diagnose pneumonia.

Aim: To investigate the accuracy of lung ultrasound compared to CXR for the diagnosis of pneumonia children by meta-analysis method.

Material and methods: Original articles which evaluated accuracy of lung ultrasound compared to chest X-ray for the diagnosis of pneumonia in children, published between 1 January 2010 and 20 March 2021, were identified in the PubMed, Web of Science, Embase, Scopus and Cochrane Library databases. Data synthesis and statistical analysis were carried out using STATA software. Odds ratios with 95\% confidence interval (CI), fixed effect model and mean difference with $95 \% \mathrm{Cl}$, random effect model (REM) were calculated.

Results: At the first step, 1016 potentially important research abstracts and titles were discovered in our electronic searches, 8 papers were in agreement with our inclusion criteria. The statistical analysis showed sensitivity of $95.5 \%$ (95\% Cl: $93.6-97.1 \%)$ and specificity of $96.3 \%$ (95\% Cl: 92.1-98.4\%) for the lung ultrasound, and CXR sensitivity and specificity were $87.4 \%$ (95\% Cl: $84.3-90.0 \%)$ and $98.6 \%$ (95\% Cl: $95.8-99.6 \%)$, respectively.

Conclusions: The present study showed that ultrasound can be useful in diagnosing pneumonia in children, and due to the lack of risk of receiving radiation, this method is even more preferred.
\end{abstract}

Key words: asthma, lung ultrasound, chest X-ray, pneumonia.

\section{Introduction}

Pneumonia is an infectious disease of the lower respiratory tract in children with high mortality worldwide, affecting more than 150 million children and the reason of approximately 3 million deaths of children under 5 years [1, 2]. It is often accompanied by cough, fever, pleuritic chest pain, and shortness of breath. Pneumonia can develop to respiratory failure, cardiac arrhythmias and kidney failure. The prevalence of this disease is 12 per 1000 people [3]. Viral and bacterial pneumonia are usually associated with a higher respiratory tract virus [4, 5]. For children with suspected pneumonia, the diagnosis requires a chest $X$-ray (CXR) with a lateral view [6]. Pneumonia in children can be classified according to different criteria. These classifications are fully applicable to decide on the management and treatment of the disease. Pneumonia is divided into different types based on the origin of the infection, microbial agent, clinical process, site of involvement, severity of the disease and the presence or absence of accompanying complications [7-12]. Based on the clinical course, typical pneumonia is usually seen with a sudden onset of high fever and local infiltration in the chest image, is caused by common bacteria such as pneumococcus and Haemophilus influenzae and responds to treatment with antibiotics. Also, atypical pneumonia in which this type of pneumonia usually has a gradual course, the fever is usually not high and the lung lesions on the chest are diffuse and are caused

Address for correspondence: Xiaoyun Dong, Department of Ultrasound, Qilu Hospital (Qingdao), Cheeloo College of Medicine, Shandong University, 758 Hefei Road, Qingdao, Shandong 266035, China, e-mail: xiaoyun.dong@protonmail.com Received: 8.05.2021, accepted: 24.05.2021. 
by common non-bacterial agents such as viruses, Mycobacterium fungi, Mycoplasma, chlamydia, etc. also, does not respond well to treatment with beta-lactam antibiotics [13-15]. As mentioned, infectious agents such as bacterial, viral, protozoal, etc. are the causes of pneumonia. However, in studies, about $25-33 \%$ of the infectious agents causing pneumonia have not been identified or in some cases, pneumonia with two microbes has been seen simultaneously, so that about $41 \%$ of patients hospitalized due to pneumonia of two or more microorganisms as their etiological factor have been identified [16-18]. Basically, after the diagnosis of pneumonia in children, it is usually divided into three categories: bacterial, viral, and atypical pneumonia based on the sum of the four findings of sub-pneumonia: a) clinical findings, b) epidemiological findings, c) radiological findings and d) initial laboratory findings such as erythrocyte sedimentation rate (ESR), complete blood count (CBC) and C-reactive protein (CRP) [19-21]. Although the diagnosis of pneumonia is usually clinical, a simple CXR can help make the diagnosis more definitive. Radiographic findings in younger children are not directly related to clinical findings. As severe pneumonia may be seen on a CXR without obvious auditory symptoms, an X-ray of the lungs is recommended in infants, especially those under 6 months of age, if there is any diagnostic doubt [22, 23]. The profile picture usually provides more information on the improvement of abnormal radiographic findings than the improvement of the patient's clinical findings with a delay of several weeks to several months, so that usually 4 to 6 weeks later the lung picture becomes normal. However, an abnormal lung picture after 3 months of pneumonia in a child will need further examination. Therefore, delayed lung imaging is not recommended for routine follow-up of children with acute pneumonia unless absolutely necessary [24, 25]. According to international guidelines, CXR is not usually required in all children with mild clinical symptoms and CAP symptoms, but only in severe cases requires hospitalization or in cases of refractory treatment. In addition, lateral CXR was not always performed. Blurring of the junction in $\mathrm{X}$-rays is considered a radiological feature of pneumonia. It is also confirmed by interstitial influences, which are considered evidence of pneumonia [26, 27]. There are two major disadvantages of using CXR to diagnose pneumonia in children: first, exposure to radiation findings. Current data show that the risk of fatal cancer in children due to radiation exposure is not negligible as there is high life expectancy and high tissue radiosensitivity. And second, initiating treatment without confirmation of diagnosis or knowledge of the underlying pathology, accidentally leads to overuse of antibiotics. Therefore, alternative free diagnostic tools should be used when assessing CAP in children, and lung ultrasound (LUS) offers this advantage $[28,29]$. LUS can be used for the initial analysis of the complications of pneumonia such as pleu- ral effusion. Some researchers reported that ultrasound was used to analyse pneumonia with $93.4 \%$ sensitivity and specificity of $97.7 \%$ [3]. Also, less regulatory requirements, relatively lower costs, and immediate bedside results are some of other advantages of LUS.

\section{Aim}

Given the importance of accurate diagnosis of pneumonia as well as the disadvantages of using chest radiography, and documentary evidence of the ability of ultrasound to diagnose pneumonia in children, the object of the current study is to investigate the accuracy of lung ultrasound compared to CXR for the diagnosis of children with asthma and pneumonia by meta-analysis method.

\section{Material and methods}

\section{Search method}

This systematic review and meta-analysis study were conducted in 2021, using the International Standard of Preferred Reporting Items for Systematic Reviews and Meta-Analyses (PRISMA). The original articles which evaluated accuracy of LUS compared to CXR for the diagnosis of pneumonia in children, published between 1 January 2010 and 20 March 2021, were identified in the PubMed, Web of Science, Embase, Scopus and Cochrane Library databases. Data synthesis and statistical analysis were carried out using STATA software. Odds ratios with $95 \%$ confidence interval (CI), fixed effect model and Mantel-Haenszel method and mean difference with 95\% Cl, random effect model and REML were calculated.

PubMed searching was performed using MeSH terms: ("Lung/diagnosis"[MeSH] OR "Lung/detection"[MeSH]) OR ("Respiratory /diagnosis"[MeSH] OR "Respiratory/ detection"[MeSH]) AND "CXR, children"[MeSH]) AND "ultrasonography, children"[MeSH]).

In other databases the following keywords were used to search: "ultrasonography" or "ultrasound" and "pneumonia" and "chest X-ray" or "CXR" and "children" or "childhood" or "paediatric".

Inclusion criteria included were prospective and retrospective studies and studies which examined patients only at paediatric departments and not in the emergency wards. Case studies, case reports and reviews and studies without the control group were excluded from the present study.

\section{Data extraction methods}

Data extracted from the studies included study design, sample size, mean age, stage, follow-up and treatment duration. Cochrane Collaboration's tool [30] was used to assess quality of the studies that were included in the present meta-analysis. The scale scores for low risk was 1 and for high and unclear risk was 0 , scale scores range from 
0 to 6 and higher score means higher quality. Hazard ratio, odds ratio and risk ratio with $95 \% \mathrm{Cl}$, fixed effect model, inverse-variance and random effects model were used to deal with potential heterogeneity and $R$ showed heterogeneity. $I^{2}$ values above $50 \%$ signified moderate-to-high heterogeneity. The meta-analysis has been evaluated with the statistical software Stata.

\section{Results}

The present study is a review of other studies, the results of which are a report of the results of the selected articles that have been presented for each required information. So, in this section we will introduce studies.

At the beginning of the study, 1017 articles were extracted from the search strategy, including 452 articles from Embase, 23 articles from the Cochrane Library, 348 articles from Pubmed, and 194 articles from the Science Citation Index Expanded. Initially, 931 articles were excluded from the study due to inaccurate title or abstract of the article. Of the other 86 articles, 57 were excluded from the study because of non-original data. Out of 29 other articles, 14 articles were excluded from the study due to being duplicate, and 7 articles excluded due to emergency department-based research. Finally, 8 articles were selected for analysis but 1 article was added after manual checking of references and this way 9 [31-39] articles were finally obtained (Figure 1).
Articles were published between 2013 to 2021 and included 4 studies performed in Italy, 1 study in Russia, 1 study in Taiwan, 1 study in Poland, 1 study in India and 1 study in Pakistan. Evaluation of the study design showed that 7 studies were prospective and 2 studies were retrospective ones. The linear, convex and micro convex probes were used in LUS. The minimum sample size was 52 and the maximum sample size was 22 and the total number of patients in 9 articles was 1124 . The age range of the patients was 0-18 years. In 6 studies LUS reported consolidations and in 1 study the results were consolidations focal B-lines (FBL), pleural line (PLA), in 1 study consolidations FBL and in the other study consolidations, false-positive (PF), FBL (Table 1).

The statistical analysis showed sensitivity of $95.5 \%$ (95\% confidence interval (Cl): 93.6-97.1\%) and specificity of $96.3 \%$ (95\% Cl: $92.1-98.4 \%)$ for the LUS, and CXR sensitivity and specificity were $87.4 \%$ (95\% Cl: 84.3-90.0\%) and $98.6 \%$ (95\% Cl: 95.8-99.6\%), respectively (Table 2, Figure 2).

\section{Discussion}

Diagnosis and treatment of children with pneumonia are still done using chest radiography, and due to the complications of $X$-rays, cost, unavailability, and possible errors, many researchers are looking for a safer alternative. Lung ultrasound has been considered by researchers as a test

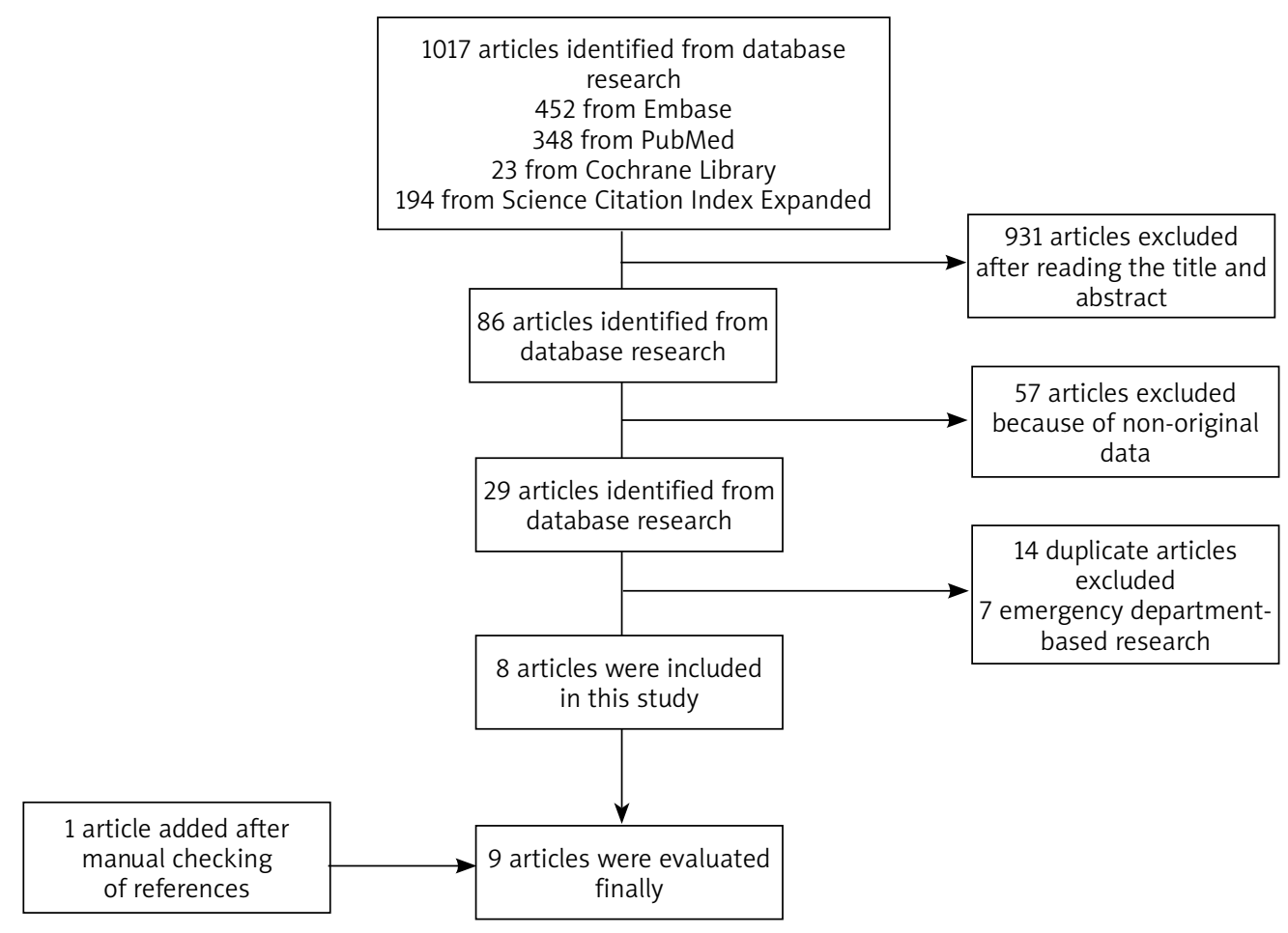

Figure 1. Flow chart of study selection 
Table 1. Characteristics of original articles included in the study

\begin{tabular}{|c|c|c|c|c|c|c|c|c|c|c|c|c|c|c|}
\hline Author & Year & Study design & Country & Age & $\begin{array}{l}\text { LUS } \\
\text { probe }\end{array}$ & $\begin{array}{l}\text { Lung } \\
\text { fields }\end{array}$ & $\begin{array}{l}\text { Diagnostic LUS } \\
\text { findings }\end{array}$ & $\begin{array}{l}\text { LUS/ } \\
\text { CXR }\end{array}$ & Patients & Pneumonia & TP & TN & FP & $\mathrm{FN}$ \\
\hline \multirow[t]{2}{*}{ Caiulo [31] } & \multirow[t]{2}{*}{2013} & \multirow[t]{2}{*}{ Prospective } & \multirow[t]{2}{*}{ Italy } & \multirow[t]{2}{*}{$1-16 y$} & \multirow[t]{2}{*}{ Linear } & \multirow[t]{2}{*}{6} & \multirow{2}{*}{$\begin{array}{l}\text { Consolidations } \\
\text { FBL, PLA }\end{array}$} & LUS & \multirow[t]{2}{*}{102} & \multirow[t]{2}{*}{89} & 88 & 13 & 0 & 1 \\
\hline & & & & & & & & CXR & & & 81 & 13 & 0 & 8 \\
\hline \multirow[t]{2}{*}{ Reali [32] } & \multirow[t]{2}{*}{2014} & \multirow[t]{2}{*}{ Prospective } & \multirow[t]{2}{*}{ Italy } & \multirow[t]{2}{*}{$0-16$ y } & \multirow[t]{2}{*}{ Linear } & \multirow[t]{2}{*}{4} & \multirow{2}{*}{$\begin{array}{c}\text { Consolidations } \\
\text { FBL }\end{array}$} & LUS & \multirow[t]{2}{*}{107} & \multirow[t]{2}{*}{81} & 76 & 25 & 1 & 5 \\
\hline & & & & & & & & CXR & & & 66 & 24 & 2 & 15 \\
\hline \multirow{2}{*}{$\begin{array}{l}\text { Dianova } \\
\text { [33] }\end{array}$} & \multirow[t]{2}{*}{2015} & \multirow[t]{2}{*}{ Prospective } & \multirow[t]{2}{*}{ Russia } & \multirow[t]{2}{*}{$0-18$ y } & \multirow{2}{*}{$\begin{array}{l}\text { Linear, } \\
\text { convex }\end{array}$} & \multirow[t]{2}{*}{6} & \multirow[t]{2}{*}{ Consolidations } & LUS & \multirow[t]{2}{*}{154} & \multirow[t]{2}{*}{154} & 147 & 0 & 0 & 7 \\
\hline & & & & & & & & CXR & & & 126 & 0 & 0 & 28 \\
\hline \multirow[t]{2}{*}{ Iorio [34] } & \multirow[t]{2}{*}{2015} & Retrospective & Italy & $0-12$ y & Linear & 6 & Consolidations & LUS & 52 & 29 & 28 & 22 & 1 & 1 \\
\hline & & & & & & & & CXR & & & 25 & 22 & 1 & 4 \\
\hline Ho [35] & 2015 & Retrospective & Taiwan & $73.2 \mathrm{~m}$ & Convex & 6 & Consolidations & LUS & 163 & 163 & 159 & 0 & 0 & 4 \\
\hline & & & & & & & & CXR & & & 151 & 0 & 0 & 12 \\
\hline Urbankowska & 2015 & Prospective & Poland & $0-18$ y & Linear & 6 & Consolidations & LUS & 106 & 106 & 71 & 0 & 5 & 30 \\
\hline & & & & & & & & CXR & & & 76 & 0 & 0 & 30 \\
\hline Guerra [37] & 2016 & Prospective & Italy & $0-16$ & Convex, & 6 & Consolidations & LUS & 222 & 222 & 207 & 0 & 7 & 8 \\
\hline & & & & & 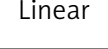 & & & CXR & & & 197 & 0 & 17 & 18 \\
\hline Yadav [38] & 2017 & Prospective & India & $0-5$ & Micro & 6 & Consolidations, & LUS & 118 & 106 & 105 & 0 & 0 & 13 \\
\hline & & & & & & & & CXR & & & 101 & 0 & 0 & 17 \\
\hline Sharif [39] & 2021 & Prospective & Pakistan & $2-12$ y & Linear & 6 & Consolidation & LUS & 100 & 64 & 64 & 0 & 0 & 36 \\
\hline & & & & & & & & CXR & & & 50 & 0 & 0 & 16 \\
\hline
\end{tabular}

CXR-chest radiography, FN-false-negative, FP-false-positive, LUS - lung ultrasound, TN-true-negative, TP-true-positive.

Table 2. Individual and cumulative (random effects model) statistics for original articles included in this study

\begin{tabular}{|c|c|c|c|c|c|}
\hline Author & LUS/ CXR & Sensitivity $(95 \% \mathrm{Cl})$ & Specificity $(95 \% \mathrm{Cl})$ & $\begin{array}{l}\text { Positive predictive } \\
\text { value }(95 \% \mathrm{Cl})\end{array}$ & $\begin{array}{l}\text { Negative predictive } \\
\text { value }(95 \% \mathrm{Cl})\end{array}$ \\
\hline \multirow[t]{2}{*}{ Caiulo [31] } & LUS & 98.9\% (93.9-100) & $100 \%(75.3-100)$ & $100 \%(95.9-100)$ & 92.9\% (66.1-99.8) \\
\hline & CXR & $91 \%(83.1-96.0)$ & $100 \%(75.3-100)$ & $100 \%(95.5-100)$ & $61.9 \%(38.4-81.9)$ \\
\hline \multirow[t]{2}{*}{ Reali [32] } & LUS & $93.8 \%(86.2-98.0)$ & $96.2 \%(80.4-99.9)$ & 98.7\% (93.0-100) & $86.2 \%(68.3-96.1)$ \\
\hline & CXR & $81.5 \%(71.3-89.2)$ & $92.3 \%(74.9-99.1)$ & $97.1 \%(89.8-99.6)$ & $61.5 \%(44.6-76.6)$ \\
\hline \multirow[t]{2}{*}{ Dianova [33] } & LUS & $95.5 \%(90.0-98.2)$ & NA & $100 \%(97.5-100)$ & $0.0 \%(0.0-41.0)$ \\
\hline & CXR & $81.8 \%(74.8-87.6)$ & NA & $100 \%(97.1-100)$ & $0.0 \%(0-12.3)$ \\
\hline \multirow[t]{2}{*}{ lorio [34] } & LUS & $96.6 \%(82.2-99.9)$ & 95.7\% (78.1-99.9) & $96.6 \%(82.2-99.9)$ & 95.7\% (78.1-99.9) \\
\hline & CXR & $86.2 \%(68.3-96.1)$ & 95.7\% (78.1-99.9) & $96.2 \%(80.4-99.9)$ & $84.6 \%(65.1-95.6)$ \\
\hline \multirow[t]{2}{*}{ Ho [35] } & LUS & $97.5 \%(93.8-99.3)$ & NA & $100 \%(97.7-100)$ & $0.0 \%(0.0-60.2)$ \\
\hline & CXR & $92.6 \%(87.5-96.1)$ & NA & $100 \%(97.0-100)$ & $0.0 \%(0.0-26.5)$ \\
\hline \multirow[t]{2}{*}{ Urbankowska [36] } & LUS & 93.0\% (85.0-98.0) & $100 \%(88.0-100)$ & $100 \%(95.9-100)$ & $0.0 \%(0.0-41.0)$ \\
\hline & CXR & $100 \%(95.0-100)$ & $100 \%(88.0-100)$ & $100 \%(95.5-100)$ & $0.0 \%(0-12.3)$ \\
\hline \multirow[t]{2}{*}{ Guerra [37] } & LUS & $97.0 \%(93.0-99.0)$ & $100 \%(63.0-100)$ & $100 \%(95.9-100)$ & $0.0 \%(0.0-41.0)$ \\
\hline & CXR & $92 \%(88.0-95.0)$ & $100 \%(63.0-100)$ & $100 \%(95.5-100)$ & $0.0 \%(0-12.3)$ \\
\hline \multirow[t]{2}{*}{ Yadav [38] } & LUS & $89.0 \%$ (81.9-94.0) & NA & $100 \%(96.5-100)$ & $0.0 \%(0.0-24.7)$ \\
\hline & CXR & 85.6\% (77.9-91.4) & NA & $100 \%(96.4-100)$ & $0.0 \%(0.0-19.5)$ \\
\hline \multirow[t]{2}{*}{ Sharif [39] } & LUS & $100 \%(93.0-100)$ & $92.3 \%$ (63.0-99.9) & 95.31\% (95.9-99.9) & $100 \%(78.1-100)$ \\
\hline & CXR & $81.96 \%$ (80.0-95.0) & $100 \%(63.0-100)$ & $100 \%(95.5-100)$ & $78.0 \%(68.3-96.1)$ \\
\hline \multirow[t]{2}{*}{ Total } & LUS & $95.5 \%(93.6-97.1)$ & $96.3 \%(92.1-98.4)$ & $99.0 \%$ (97.8-99.8) & $63.6 \%(40.7-82.7)$ \\
\hline & CXR & $87.4 \%$ (84.3-90.0) & 98.6\% (95.8-99.6) & 99.5\% (99.1-99.9) & $44.6 \%(20.4-68.3)$ \\
\hline
\end{tabular}

CXR - chest radiography, LUS - lung ultrasound. 
A

$\quad$ CXR sensitivity
$\rightarrow$ Caiulo
$\rightarrow$ - Reali
$\rightarrow-$ Dianova
$\rightarrow-$ lorio
$-\because$ Ho
$\rightarrow-$ Urbankowska
$\rightarrow-$ Guerra
$\rightarrow-$ Yadav
$\rightarrow-$ Sharif
$\rightarrow-$ Total

C

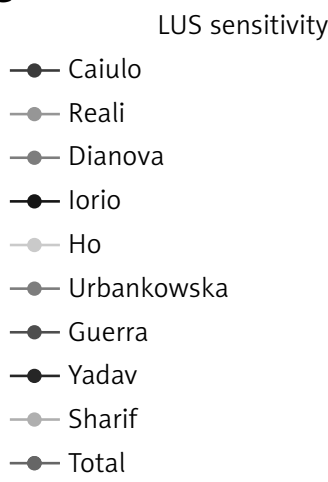

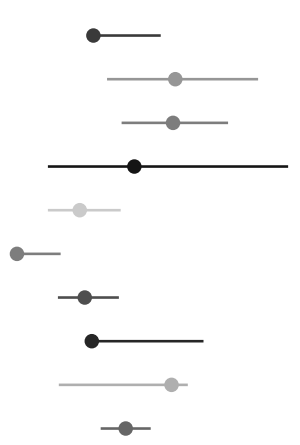

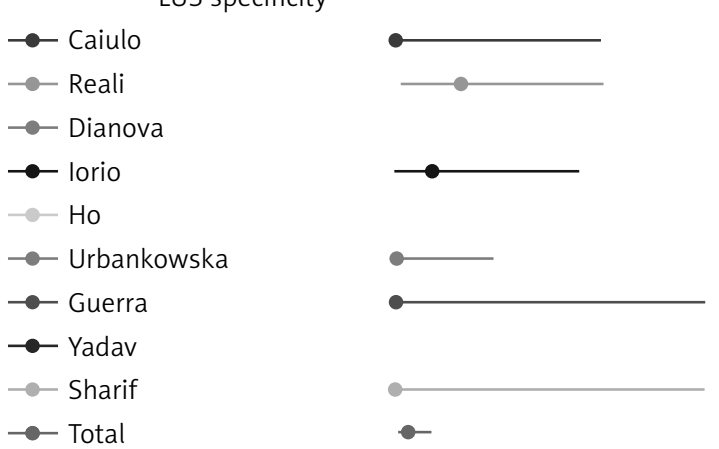

D

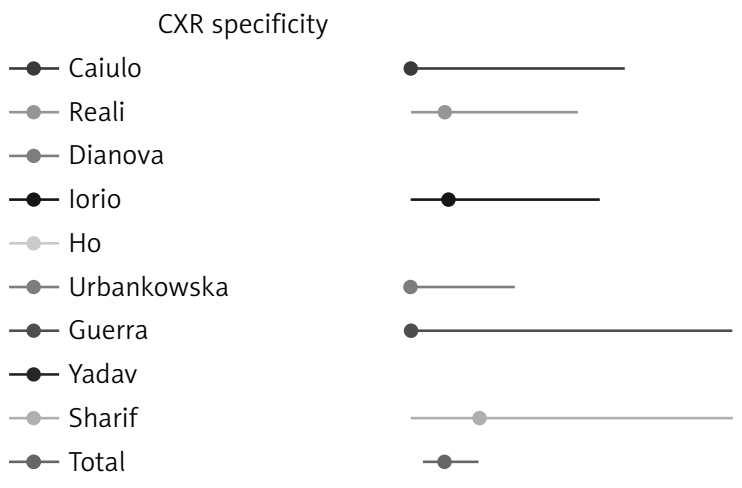

Figure 2. Forest plots: A - CXR sensitivity, B - CXR specificity, C - LUS sensitivity, D - LUS specificity

with high sensitivity and specificity and many studies have been conducted in different areas to compare these two methods. Because the results of the research were contradictory, in this study we tried to announce a final result on the present topic by reviewing all the studies done and choosing the right powerful studies. What was found in the present study showed that the sensitivity of ultrasound is higher than chest radiography but the specificity of radiography is higher than ultrasonography. The studies also showed high sensitivities of ultrasound and the researchers suggested that ultrasound can be used as a suitable tool to diagnose and continue patient management. In the study of Caiulo et al. [31], LUS was positive for the diagnosis of pneumonia in 88/89 patients, whereas CXR was positive in 81/89. Also, Reali et al. [32] reported that ultrasound had a sensitivity of $94 \%$ and specificity of $96 \%$, while CXR showed a sensitivity of $82 \%$ and a specificity of $94 \%$. Another study conducted by Dianova and Safonov [33] showed that chest ultrasound is an informative radiologically safe technique for diagnosing and dynamic control of pneumonia in children. Iorio et al. [34] also mentioned that LUS had a sensitivity of $96.5 \%$ and specificity of $95.6 \%$. The other powerful study conducted by Ho et al. [35] reported that LUS is a sensitive diagnostic tool to identify pneumonia in children. Urbankowska et al. [36] also reported that LUS had a sensitivity of 93.4\% and specificity of $100 \%$. Guerra et al. [37] showed LUS can be adopted by the clinician as a non-invasive bedside tool to expand the physical evaluation of febrile children with respiratory distress. Another study conducted by Yadav et al. [38] showed LUS can be considered to be used first before radiography in children with suspected CAP. Finally, the newest study by Sharif et al. [39] reported that LUS had a sensitivity of $100 \%$ and specificity of $92.30 \%$, but as mentioned in the result section, the total statistical analysis showed sensitivity of 95.5\% (95\% Cl: 93.6-97.1\%) and specificity of $96.3 \%$ (95\% Cl: $92.1-98.4 \%)$ for LUS, and CXR sensitivity and specificity were $87.4 \%(95 \% \mathrm{Cl}$ : 84.3-90.0\%) and 98.6\% (95\% Cl: 95.8-99.6\%), respectively.

\section{Conclusions}

Our study suggests that LUS is the best alternative to CXR and this device is a reliable and valuable tool with 
acceptable sensitivity and specificity so in conclusion, ultrasound can be useful in diagnosing children with asthma and pneumonia, and due to the lack of any risk of receiving radiation, this method is even more preferred.

\section{Conflict of interest}

The authors declare no conflict of interest.

\section{References}

1. ur Rehman A, Hassali MA, Abbas S, et al. Pharmacological and non-pharmacological management of COPD; limitations and future prospects: a review of current literature. J Public Health 2020; 28: 357-66.

2. Sheam MM, Syed SB, Nain Z, et al. Community-acquired pneumonia: aetiology, antibiotic resistance and prospects of phage therapy. J Chemother 2020; 32: 395-410.

3. Rahmati MB, Ahmadi M, Malekmohamadi SH, et al. The significance of chest ultrasound and chest X-ray in the diagnosis of children clinically suspected of pneumonia. J Med Life 2015; 8 (Spec Iss 3): 50.

4. Koo HJ, Lim S, Choe J, et al. Radiographic and CT features of viral pneumonia. Radiographics 2018; 38: 719-39.

5. Wang Z, Chen X, Lu Y, et al. Clinical characteristics and therapeutic procedure for four cases with 2019 novel coronavirus pneumonia receiving combined Chinese and Western medicine treatment. Biosci Trends 2020; 14: 64-8.

6. Yan C, Hui R, Lijuan Z, Zhou Y. Lung ultrasound vs. chest $X$-ray in children with suspected pneumonia confirmed by chest computed tomography: a retrospective cohort study. Exp Ther Med 2020; 19: 1363-9.

7. Rudan I, Tomaskovic L, Boschi-Pinto C, Campbell H. Global estimate of the incidence of clinical pneumonia among children under five years of age. Bull World Health Organ 2004; 82: 895-903.

8. Asnaoui KE, Chawki Y, Idri A. Automated methods for detection and classification pneumonia based on $x$-ray images using deep learning. arXiv preprint arXiv:2003.14363. 2020 Mar 31.

9. Saraiva AA, Ferreira NM, de Sousa LL, et al. Classification of images of childhood pneumonia using convolutional neural networks. In: Proceedings of the 12th International Joint Conference on Biomedical Engineering Systems and Technologies (BIOSTEC 2019), pages 112-119.

10. Hart A, Lee EY. Pediatric chest disorders: practical imaging approach to diagnosis. In: Diseases of the Chest, Breast, Heart and Vessels 2019-2022: Diagnostic and Interventional Imaging [Internet]. Cham (CH): Springer 2019. Chapter 10.

11. De Blasio A, Chioni L, Adorni G. Differential diagnosis of types of pneumonia in the elderly. In: Covid-19 Airway Management and Ventilation Strategy for Critically III Older Patients. Vargas N, Esquinas A (eds). Springer, Cham 2020; 35-66.

12. Eslamy HK, Newman B. Pneumonia in normal and immunocompromised children: an overview and update. Radiol Clin 2011; 49: 895-920.

13. Jereb M, Kotar T. Usefulness of procalcitonin to differentiate typical from atypical community-acquired pneumonia. Wien Klin Wochenschr 2006; 118: 170-4.

14. Cunha BA. The atypical pneumonias: clinical diagnosis and importance. Clin Microbiol Infect 2006; 12: 12-24.
15. Hon KL, Leung AS, Cheung KL, et al. Typical or atypical pneumonia and severe acute respiratory symptoms in PICU. Clin Respir J 2015; 9: 366-71.

16. Lee WM, Grindle K, Pappas T, et al. High-throughput, sensitive, and accurate multiplex PCR-microsphere flow cytometry system for large-scale comprehensive detection of respiratory viruses. J Clin Microbiol 2007; 45: 2626-34.

17. Templeton KE, Scheltinga SA, Van den Eeden WCJFM, et al. Improved diagnosis of the etiology of community-acquired pneumonia with real-time polymerase chain reaction. Clin Infect Dis 2005; 41: 345-51.

18. Juven T, Mertsola J, Waris M, et al. Etiology of communityacquired pneumonia in 254 hospitalized children. Pediatr Infect Dis J 2000; 19: 293-8.

19. Koenig SM, Truwit JD. Ventilator-associated pneumonia: diagnosis, treatment, and prevention. Clin Microbiol Rev 2006; 19: 637-57.

20. Muscedere J, Dodek P, Keenan S, et al.; VAP Guidelines Committee, Canadian Critical Care Trials Group. Comprehensive evidence-based clinical practice guidelines for ventilatorassociated pneumonia: diagnosis and treatment. J Crit Care 2008; 23: 138-47.

21. Hoare Z, Lim WS. Pneumonia: update on diagnosis and management. BMJ 2006; 332: 1077-9.

22. Coote N, Craig J, Heath PT, et al. BTS guidelines for the management of community acquired pneumonia in childhood. Thorax 2002; 57: X-i24.

23. Lynch T, Platt R, Gouin S, et al. Can we predict which children with clinically suspected pneumonia will have the presence of focal infiltrates on chest radiographs? Pediatrics 2004; 113: e186-9.

24. Rigsby CK, Strife JL, Johnson ND, et al. Is the frontal radiograph alone sufficient to evaluate for pneumonia in children? Pediatr Radiol 2004; 34: 379-83.

25. Sazawal S, Black RE. Effect of pneumonia case management on mortality in neonates, infants, and preschool children: a meta-analysis of community-based trials. Lancet Infect Dis 2003; 3: 547-56.

26. Harris M, Clark J, Coote N, et al. British Thoracic Society guidelines for the management of community acquired pneumonia in children: update 2011. Thorax 2011; 66 (Suppl 2): ii1-23.

27. Cherian T, Mulholland EK, Carlin JB, et al. Standardized interpretation of paediatric chest radiographs for the diagnosis of pneumonia in epidemiological studies. Bull World Health Organ 2005; 83: 353-9.

28. Johnson J, Kline JA. Intraobserver and interobserver agreement of the interpretation of pediatric chest radiographs. Emerg Radiol 2010; 17: 285-90.

29. Alzen G, Benz-Bohm G. Radiation protection in pediatric radiology. Dtsch Arztebl Int 2011; 108: 407-14.

30. Higgins JP, Altman DG, Gøtzsche PC, et al. The Cochrane Collaboration's tool for assessing risk of bias in randomised trials. BMJ 2011; 343: d5928.

31. Caiulo VA, Gargani L, Caiulo S, et al. Lung ultrasound characteristics of communit-acquired pneumonia in hospitalized children. Pediatr Pulmonol 2013; 48: 280-7.

32. Reali F, Papa GF, Carlucci P, et al. Can lung ultrasound replace chest radiography for the diagnosis of pneumonia in hospitalized children? Respiration 2014; 88: 112-5.

33. Dianova I, Safonov DV. Ultrasound monitoring and age sonographic characteristics of community-acquired pneumonia in children. Clin Med 2015; 7: 113-8. 
34. Iorio G, Capasso M, De Luca G, et al. Lung ultrasound in the diagnosis of pneumonia in children: proposal for a new diagnostic algorithm. Peer J 2015; 3: e1374.

35. Ho MC, Ker CR, Hsu JH, et al. Usefulness of lung ultrasound in the diagnosis of community-acquired pneumonia in children. Pediatr Neonatol 2015; 56: 40-5.

36. Urbankowska E, Krenke K, Drobczyński Ł, et al. Lung ultrasound in the diagnosis and monitoring of community acquired pneumonia in children. Respir Med 2015; 109: 1207-12.

37. Guerra M, Crichiutti G, Pecile P, et al. Ultrasound detection of pneumonia in febrile children with respiratory distress: a prospective study. Eur J Pediatr 2016; 175: 163-70.

38. Yadav KK, Awasthi S, Parihar A. Lung ultrasound is comparable with chest roentgenogram for diagnosis of community-acquired pneumonia in hospitalised children. Indian J Pediatr 2017; 84: 499-504

39. Sharif M, Saeed T, Saheel K, et al. Comparison of chest Xray with lung ultrasound in the diagnosis of pneumonia in children aged 02 months to 12 years. J Rawalpindi Med Coll 2021; 25: 87-90. 